\title{
The Convergence Ball and Error Analysis of the Relaxed Secant Method
}

\author{
Rongfei Lin, ${ }^{1}$ Qingbiao Wu, ${ }^{2}$ Minhong Chen, ${ }^{3}$ and Lu Liu ${ }^{2}$ \\ ${ }^{1}$ Department of Mathematics, Taizhou University, Linhai, Zhejiang 317000, China \\ ${ }^{2}$ Department of Mathematics, Zhejiang University, Hangzhou, Zhejiang 310027, China \\ ${ }^{3}$ Department of Mathematics, Zhejiang Sci-Tech University, Hangzhou, Zhejiang 310012, China
}

Correspondence should be addressed to Qingbiao Wu; qbwu@zju.edu.cn

Received 4 November 2016; Revised 17 January 2017; Accepted 12 February 2017; Published 8 March 2017

Academic Editor: Kaliyaperumal Nakkeeran

Copyright (c) 2017 Rongfei Lin et al. This is an open access article distributed under the Creative Commons Attribution License, which permits unrestricted use, distribution, and reproduction in any medium, provided the original work is properly cited.

A relaxed secant method is proposed. Radius estimate of the convergence ball of the relaxed secant method is attained for the nonlinear equation systems with Lipschitz continuous divided differences of first order. The error estimate is also established with matched convergence order. From the radius and error estimate, the relation between the radius and the speed of convergence is discussed with parameter. At last, some numerical examples are given.

\section{Introduction}

Many scientific problems can be concluded to the form of nonlinear systems. Finding the solutions of nonlinear systems is widely required in both mathematical physics and nonlinear dynamical systems. In this paper, we will establish the convergence ball and error analysis of the relaxed secant method of nonlinear systems. Consider

$$
F(x)=0,
$$

where $F$ is a nonlinear operator defined on a convex subset $\Omega$ of a Banach space $X$ with values in another Banach space $Y$. When $F$ is nonlinear, iterative methods are generally adopted to solve the system:

$$
x_{n+1}=\Psi\left(x_{n}\right), \quad x_{0} \text { is given } .
$$

The most widely used iterative method is Newton's method which can be described as

$$
x_{n+1}=x_{n}-F\left(x_{n}\right)^{-1} F\left(x_{n}\right), \quad x_{0} \text { is given. }
$$

This method and Newton-like methods have been studied well by many authors (see [1-12]).

Newton's method requires that $F$ is differentiable. Thus, when $F$ is nondifferentiable, Newton method cannot be applied on it. We have to turn to other methods that do not need to evaluate derivatives. In their algorithms, instead of derivatives, divided differences are always used. The classical method of this type is the secant method.

Let $\Phi(X, Y)$ denote the space of the bounded linear maps from $X$ to $Y$. If the following equality holds,

$$
[X, Y ; F](x-y)=F(x)-F(y),
$$

then, we call the operator $[X, Y ; F] \in \Phi(X, Y)$, at the points $x$ and $y(x \neq y)$, a divided difference of order one of the nonlinear operator $F$.

By the above definition, secant method can be generalized to Banach spaces, it is described as the following scheme:

$$
\begin{array}{r}
x_{n+1}=x_{n}-\left[x_{n-1}, x_{n} ; F\right]^{-1} F\left(x_{n}\right), \\
(n>0) x_{0}, x_{-1} \in \Omega .
\end{array}
$$

An interesting issue here is to estimate the radius of the convergence ball of an iterative method. Suppose $x_{*}$ is a solution of the nonlinear system (1). Denote with $B\left(x_{*}, r\right) \subset$ $X$ an open ball with center $x_{*}$ and radius $r$. The open ball $B\left(x_{*}, r\right) \subset X$ is called a convergence ball of an iteration, if the sequence generated by the iterative method converges with any initial value in the ball. Under the assumption that the 
nonlinear operator $F$ has Fréchet derivatives satisfying the Hölder condition,

$$
\begin{aligned}
& \left\|F^{\prime}\left(x_{*}\right)^{-1}\left([x, y ; F]-F^{\prime}(z)\right)\right\| \\
& \leqslant K\left(\|x-z\|^{p}+\|y-z\|^{p}\right), \\
& \forall x, y, z \in \Omega \text { for some } K>0 .
\end{aligned}
$$

Ren and $\mathrm{Wu}[13]$ have given the radius of the convergence ball which is $r_{p}=\sqrt[p]{(1+p) / K(1+2 p)}$.

The convergence ball, the semilocal convergence of secant method, and secant-like method have been studied by many other authors (see [13-18]). In this paper, similar to the relaxed Newton's method in [7], we considered the relaxed secant method which can be written as the following form:

$$
x_{n+1}=x_{n}-\lambda\left[x_{n-1}, x_{n} ; F\right]^{-1} F\left(x_{n}\right), \quad x_{0}, x_{-1} \in \Omega ;
$$

here, $\lambda \in(0,2)$ is called the relaxed parameter. When $\lambda=1$, it will be the normal secant method.

In this paper, we will study the convergence ball of (7) under the assumption that the nonlinear operator $F$ has Fréchet derivatives satisfying the following Lipschitz condition:

$$
\begin{aligned}
& \left\|F^{\prime}\left(x_{*}\right)^{-1}([x, y ; F]-[v, w ; F])\right\| \\
& \leqslant K(\|x-v\|+\|y-w\|),
\end{aligned}
$$

$$
\forall x, y, v, w \in \Omega \text { for some } K>0 .
$$

Under the Lipschitz condition, the radius $r_{\lambda_{1}}$ of the relaxed method is proved to be $\lambda / 4 K$ when $0<\lambda \leqslant 1$; and the radius $r_{\lambda_{2}}$ of the relaxed method is proved to be $(2-\lambda) / 4 K \lambda$ when $1<\lambda<2$. The error estimate is also given.

\section{Convergence Ball}

Theorem 1. Suppose $F\left(x_{*}\right)=0$, where the nonlinear operator $F$ is Fréchet differentiable on $\Omega, F^{\prime}\left(x_{*}\right)^{-1}$ exists, the Lipschitz condition (8) holds, and $0<\lambda<2$. Denote

$$
\begin{aligned}
& r_{\lambda_{1}}=\frac{\lambda}{4 K}, \\
& r_{\lambda_{2}}=\frac{(2-\lambda)}{4 K \lambda} .
\end{aligned}
$$

When $0<\lambda \leqslant 1$, starting from any two initial points $x_{0}, x_{-1}$ in ball $B\left(x_{*}, r_{\lambda_{1}}\right)$, the sequence $\left\{x_{n}\right\}$ generated by the relaxed secant method (7) converges to the solution $x_{*}$. When $1<\lambda<$ 2 , the sequence $\left\{x_{n}\right\}$ generated by the relaxed secant method (7) converges to the solution $x_{*}$, with any two initial points $x_{0}, x_{-1}$ in ball $B\left(x_{*}, r_{\lambda_{2}}\right) . x_{*}$ is the unique solution in ball $B\left(x_{*}, 1 / K\right)$, that is bigger than ball $B\left(x_{*}, r_{\lambda_{1}}\right)$ and ball $B\left(x_{*}, r_{\lambda_{2}}\right)$. Moreover, we have the following error estimate:

$$
\begin{aligned}
& \left\|x_{n}-x_{*}\right\| \leqslant\left(\frac{2-\lambda}{1-2 K \theta}-1\right)^{n}, \quad \text { if } 0<\lambda \leqslant 1, \\
& \left\|x_{n}-x_{*}\right\| \leqslant\left(\frac{(4 \lambda-2) K \theta+\lambda-1}{1-2 K \theta}\right)^{n}, \quad \text { if } 1<\lambda<2,
\end{aligned}
$$

where $\theta=\max \left\{\left\|x_{0}-x_{*}\right\|,\left\|x_{-1}-x_{*}\right\|\right\}$.
Proof. We will prove the above theorem by induction. Firstly, when $0<\lambda<2$, by Lipschitz condition, it is easy to get

$$
\begin{aligned}
\| I & -F^{\prime}\left(x_{*}\right)^{-1}\left[x_{-1}, x_{0} ; F\right] \| \\
& =\left\|F^{\prime}\left(x_{*}\right)^{-1}\left(F^{\prime}\left(x_{*}\right)-\left[x_{-1}, x_{0} ; F\right]\right)\right\| \\
& \leqslant K\left(\left\|x_{-1}-x_{*}\right\|+\left\|x_{0}-x_{*}\right\|\right)<1 .
\end{aligned}
$$

By Banach lemma, we can know $\left[x_{-1}, x_{0} ; F\right]$ is invertible. Since $x_{1}$ is well defined and

$$
\begin{aligned}
& \left\|\left(F^{\prime}\left(x_{*}\right)^{-1}\left[x_{-1}, x_{0} ; F\right]\right)^{-1}\right\| \\
& \leqslant \frac{1}{1-K\left(\left\|x_{-1}-x_{*}\right\|+\left\|x_{0}-x_{*}\right\|\right)},
\end{aligned}
$$

we can conduct

$$
\begin{aligned}
& \left\|F^{\prime}\left(x_{*}\right)^{-1}\left[x_{-1}, x_{0} ; F\right]\right\| \\
& \quad=\left\|F^{\prime}\left(x_{*}\right)^{-1}\left(\left[x_{-1}, x_{0} ; F\right]-F^{\prime}\left(x_{*}\right)+F^{\prime}\left(x_{*}\right)\right)\right\| \\
& \quad \leqslant K\left(\left\|x_{-1}-x_{*}\right\|+\left\|x_{0}-x_{*}\right\|\right)+1 .
\end{aligned}
$$

Then, we can give the estimate of $\left\|x_{1}-x_{*}\right\|$ when $0<\lambda \leqslant$ 1. From $F\left(x_{*}\right)=0$, we have

$$
\begin{aligned}
& \left\|x_{1}-x_{*}\right\|=\left\|x_{0}-x_{*}-\lambda\left[x_{-1}, x_{0} ; F\right]^{-1} F\left(x_{0}\right)\right\| \\
& =\|\left(F^{\prime}\left(x_{*}\right)^{-1}\left[x_{-1}, x_{0} ; F\right]\right)^{-1} F^{\prime}\left(x_{*}\right)^{-1} \\
& \cdot\left(\left[x_{-1}, x_{0} ; F\right]\left(x_{0}-x_{*}\right)-\lambda\left(F\left(x_{0}\right)-F\left(x_{*}\right)\right)\right) \| \\
& \leqslant\left\|\left(F^{\prime}\left(x_{*}\right)^{-1}\left[x_{-1}, x_{0} ; F\right]\right)^{-1}\right\| \| F^{\prime}\left(x_{*}\right)^{-1} \\
& \cdot\left(\left[x_{-1}, x_{0} ; F\right]\left(x_{0}-x_{*}\right)\right) \\
& -\lambda \int_{0}^{1} F^{\prime}\left(t x_{0}+(1-t) x_{*}\right) \mathrm{d} t\left(x_{0}-x_{*}\right) \| \\
& \leqslant\left\|\left(F^{\prime}\left(x_{*}\right)^{-1}\left[x_{-1}, x_{0} ; F\right]\right)^{-1}\right\|\left\|x_{0}-x_{*}\right\| \\
& \cdot\left(\lambda \| F^{\prime}\left(x_{*}\right)^{-1}\right. \\
& -\left(\left[x_{-1}, x_{0} ; F\right]-\int_{0}^{1} F^{\prime}\left(t x_{0}+(1-t) x_{*}\right) \mathrm{d} t\right) \|+(1 \\
& \\
& \left.-\lambda F^{\prime}\left(x_{*}\right)^{-1}\left[x_{-1}, x_{0} ; F\right] \|\right) \cdot
\end{aligned}
$$


Using Lipschitz condition with (12) and (13), we have

$$
\begin{aligned}
& \left\|x_{1}-x_{*}\right\| \leqslant \frac{r_{\lambda_{1}}}{1-2 K r_{\lambda_{1}}}\left(\int _ { 0 } ^ { 1 } \lambda K \left(\left\|x_{-1}-t x_{0}-(1-t) x_{*}\right\|\right.\right. \\
& \left.+\left\|x_{0}-t x_{0}-(1-t) x_{*}\right\|\right) \mathrm{d} t+(1-\lambda)\left(K \left(\left\|x_{-1}-x_{*}\right\|\right.\right. \\
& \left.\left.\left.\quad+\left\|x_{0}+x_{*}\right\|\right)+1\right)\right) \\
& \quad=\frac{r_{\lambda_{1}}}{1-2 K r_{\lambda_{1}}}\left(\int _ { 0 } ^ { 1 } \lambda K \left(\left\|t\left(x_{-1}-x_{0}\right)+(1-t)\left(x_{-1}-x_{*}\right)\right\|\right.\right. \\
& \left.+(1-t)\left\|x_{0}-x_{*}\right\|\right) \mathrm{d} t+(1-\lambda)\left(K \left(\left\|x_{-1}-x_{*}\right\|\right.\right. \\
& \left.\left.\left.\quad+\left\|x_{0}+x_{*}\right\|\right)+1\right)\right) .
\end{aligned}
$$

Obviously, we have

$$
\left\|x_{-1}-x_{0}\right\| \leqslant\left\|x_{0}-x_{*}\right\|+\left\|x_{-1}-x_{*}\right\|
$$

From $x_{-1}, x_{0} \in B\left(x_{*}, r_{\lambda_{1}}\right)$, together with (15), (16), and $r_{\lambda_{1}}=$ $\lambda / 4 K$, we have

$$
\begin{aligned}
& \left\|x_{1}-x_{*}\right\| \\
& \quad<\frac{r_{\lambda_{1}}}{1-2 K r_{\lambda_{1}}}\left(2 K \lambda r_{\lambda_{1}}+(1-\lambda)\left(2 K r_{\lambda_{1}}+1\right)\right) \\
& \quad=r_{\lambda_{1}} .
\end{aligned}
$$

This means $x_{1} \in B\left(x_{*}, r_{\lambda_{1}}\right)$

Similar to the procession above, when $1<\lambda<2$, we can get that

$$
\begin{aligned}
& \left\|x_{1}-x_{*}\right\|=\left\|x_{0}-x_{*}-\lambda\left[x_{-1}, x_{0} ; F\right]^{-1} F\left(x_{0}\right)\right\| \\
& \leqslant\left\|\left(F^{\prime}\left(x_{*}\right)^{-1}\left[x_{-1}, x_{0} ; F\right]\right)^{-1}\right\|\left\|x_{0}-x_{*}\right\| \\
& .\left(\lambda \| F^{\prime}\left(x_{*}\right)\right. \\
& \cdot\left(\left[x_{-1}, x_{0} ; F\right]-\int_{0}^{1} F^{\prime}\left(t x_{0}+(1-t) x_{*}\right) \mathrm{d} t\right) \|+(\lambda \\
& \left.\quad-1)\left\|F^{\prime}\left(x_{*}\right)^{-1}\left[x_{-1}, x_{0} ; F\right]\right\|\right) .
\end{aligned}
$$

By (13) and (18) and Lipschitz condition we can get

$$
\begin{aligned}
& \left\|x_{1}-x_{*}\right\| \leq \frac{r_{\lambda_{2}}}{1-2 K r_{\lambda_{2}}}\left(\int _ { 0 } ^ { 1 } \lambda K \left(\left\|x_{-1}-t x_{0}-(1-t) x_{*}\right\|\right.\right. \\
& \left.+\left\|x_{0}-t x_{0}-(1-t) x_{*}\right\|\right) \mathrm{d} t+(\lambda-1)\left(K \left(\left\|x_{-1}-x_{*}\right\|\right.\right. \\
& \left.\left.\left.\quad+\left\|x_{0}-x_{*}\right\|\right)+1\right)\right)
\end{aligned}
$$

$$
\begin{aligned}
& =\frac{r_{\lambda_{2}}}{1-2 K r_{\lambda_{2}}}\left(\int _ { 0 } ^ { 1 } \lambda K \left(\left\|t\left(x_{-1}-x_{0}\right)+(1-t)\left(x_{-1}-x_{*}\right)\right\|\right.\right. \\
& \left.+(1-t)\left\|x_{0}-x_{*}\right\|\right) \mathrm{d} t+(\lambda-1)\left(K \left(\left\|x_{-1}-x_{*}\right\|\right.\right. \\
& \left.\left.\left.+\left\|x_{0}-x_{*}\right\|\right)+1\right)\right) \leqslant \frac{r_{\lambda_{2}}}{1-2 K \lambda_{2}}\left(2 K \lambda r_{\lambda_{2}}+(\lambda-1)\left(2 K r_{\lambda_{2}}\right.\right. \\
& +1)) .
\end{aligned}
$$

For $x_{-1}, x_{0} \in B\left(x_{*}, r_{\lambda_{2}}\right)$,

$$
\begin{aligned}
& \left\|x_{1}-x_{*}\right\| \\
& \quad \leqslant \frac{K r_{\lambda_{2}}}{1-2 K \lambda_{2}}\left(2 K \lambda r_{\lambda_{2}}+(\lambda-1)\left(2 K r_{\lambda_{2}}+1\right)\right) \\
& \quad=r_{\lambda_{2}} .
\end{aligned}
$$

This means that $x_{1} \in B\left(x_{*}, r_{\lambda_{2}}\right)$ when $1<\lambda<2$.

Now, suppose $\left\{x_{k}\right\}(k=1,2, \ldots, n)$ is well defined, $x_{k} \in$ $B\left(x_{*}, r_{\lambda_{1}}\right)$, when $0<\lambda \leqslant 1 ;\left\{x_{k}\right\}(k=1,2, \ldots, n)$ is well defined, $x_{k} \in B\left(x_{*}, r_{\lambda_{2}}\right)$, when $1<\lambda<2$. Similar to the argumentation about $x_{-1}$ and $x_{0}$, when $0<\lambda<2$,

$$
\begin{aligned}
\| I & -F^{\prime}\left(x_{*}\right)^{-1}\left[x_{n-1}, x_{n} ; F\right] \| \\
& \leqslant K\left(\left\|x_{n-1}-x_{*}\right\|+\left\|x_{n}-x_{*}\right\|\right)<1 .
\end{aligned}
$$

By the Banach lemma, it is obviously known that $\left[x_{n-1}, x_{n} ; F\right]$ is invertible. Hence, $x_{n+1}$ is well defined. We also get

$$
\begin{aligned}
& \left\|\left(F^{\prime}\left(x_{*}\right)^{-1}\left[x_{n-1}, x_{n} ; F\right]\right)^{-1}\right\| \\
& \quad \leq \frac{1}{1-K\left(\left\|x_{n-1}-x_{*}\right\|+\left\|x_{n}-x_{*}\right\|\right)} .
\end{aligned}
$$

When $0<\lambda \leqslant 1$,

$$
\begin{aligned}
& \left\|x_{n+1}-x_{*}\right\| \leqslant \frac{\left\|x_{n}-x_{*}\right\|}{1-K\left(\left\|x_{n}-x_{*}\right\|+\left\|x_{n-1}-x_{*}\right\|\right)} \\
& \quad \times\left(\lambda K \int _ { 0 } ^ { 1 } \left(\left\|t\left(x_{n-1}-x_{n}\right)+(1-t)\left(x_{n-1}-x_{*}\right)\right\|\right.\right. \\
& \left.\quad+(1-t)\left\|x_{n}-x_{*}\right\|\right) \mathrm{d} t+(1-\lambda) \\
& \left.\cdot\left(K\left(\left\|x_{n-1}-x_{*}\right\|+\left\|x_{n}-x_{*}\right\|\right)+1\right)\right) .
\end{aligned}
$$

And when $1<\lambda<2$, we have

$$
\begin{aligned}
& \left\|x_{n+1}-x_{*}\right\| \leqslant \frac{\left\|x_{n}-x_{*}\right\|}{1-K\left(\left\|x_{n}-x_{*}\right\|+\left\|x_{n-1}-x_{*}\right\|\right)} \\
& \quad \times\left(\lambda K \int _ { 0 } ^ { 1 } \left(\left\|t\left(x_{n-1}-x_{n}\right)+(1-t)\left(x_{n-1}-x_{*}\right)\right\|\right.\right. \\
& \left.+(1-t)\left\|x_{n}-x_{*}\right\|\right) \mathrm{d} t+(\lambda-1) \\
& \left.\cdot\left(K\left(\left\|x_{n-1}-x_{*}\right\|+\left\|x_{n}-x_{*}\right\|\right)+1\right)\right) .
\end{aligned}
$$


By the assumptions that $x_{n-1}, x_{n} \in B\left(x_{*}, r_{\lambda_{1}}\right)$ when $0<\lambda \leqslant 1$ and $x_{n-1}, x_{n} \in B\left(x_{*}, r_{\lambda_{2}}\right)$ when $1<\lambda<2$, similar to the discussions about $x_{1}$, it is known that $x_{n+1} \in B\left(x_{*}, r_{\lambda_{1}}\right)$ when $0<\lambda \leqslant 1$ and $x_{n+1} \in B\left(x_{*}, r_{\lambda_{2}}\right)$ when $1<\lambda<2$.

Therefore, starting from any two initial points $x_{-1}, x_{0}, x_{n}$, the sequence $\left\{x_{n}\right\}$, generated by the relaxed secant method, is well defined when $0<\lambda \leqslant 1, x_{n} \in B\left(x_{*}, r_{\lambda_{1}}\right)$, and when $1<\lambda<2, x_{n} \in B\left(x_{*}, r_{\lambda_{2}}\right)$. It means that the following holds:

$$
\begin{array}{ll}
\left\|x_{n}-x_{*}\right\|<r_{\lambda_{1}}, \quad(0<\lambda \leqslant 1, n \geq-1), \\
\left\|x_{n}-x_{*}\right\|<r_{\lambda_{2}}, \quad(1<\lambda<2, n \geq-1) .
\end{array}
$$

Denote

$$
\begin{aligned}
\theta_{n} & =\left\|x_{n}-x_{*}\right\|, \\
\theta & =\max \left\{\theta_{0}, \theta_{-1}\right\} .
\end{aligned}
$$

When $0<\lambda \leqslant 1$, from (14) we can get

$$
\begin{aligned}
& \left\|x_{n+1}-x_{*}\right\| \leqslant \frac{\left\|x_{n}-x_{*}\right\|}{1-K\left(\left\|x_{n}-x_{*}\right\|+\left\|x_{n-1}-x_{*}\right\|\right)} \\
& \quad \times\left(\lambda K\left(\left\|x_{n}-x_{*}\right\|+\left\|x_{n-1}-x_{*}\right\|\right)\right. \\
& \left.\quad+(1-\lambda)\left(K\left(\left\|x_{n}-x_{*}\right\|+\left\|x_{n-1}-x_{*}\right\|\right)+1\right)\right) .
\end{aligned}
$$

Then, by (27), we have

$$
\begin{aligned}
& \theta_{n+1} \leqslant \frac{\theta_{n}}{1-K\left(\theta_{n}+\theta_{n-1}\right)}\left(\lambda K\left(\theta_{n}+\theta_{n-1}\right)\right. \\
&\left.+(1-\lambda)\left(K\left(\theta_{n}+\theta_{n-1}\right)+1\right)\right) .
\end{aligned}
$$

By (23), we know $\theta_{n}<r_{\lambda_{1}}$ for all $n$. Then by (29) and (30), we can induct

$$
\theta_{n+1}<\theta_{n}<\theta_{n-1}<\cdots<\theta_{1}<\theta
$$

Then we can see

$$
\begin{aligned}
\theta_{n+1} & \leqslant \frac{\theta_{n}}{1-K\left(\theta_{n}+\theta_{n-1}\right)}\left(K\left(\theta_{n}+\theta_{n-1}\right)+1-\lambda\right) \\
& =\theta_{n} \frac{K\left(\theta_{n}+\theta_{n-1}\right)-1+2-\lambda}{1-K\left(\theta_{n}+\theta_{n-1}\right)} \\
& =\theta_{n}\left(\frac{2-\lambda}{1-K\left(\theta_{n}+\theta_{n-1}\right)}-1\right) \\
& <\theta_{n}\left(\frac{2-\lambda}{1-2 K \theta}-1\right)<\theta\left(\frac{2-\lambda}{1-2 K \theta}-1\right)^{n+1} .
\end{aligned}
$$

Obviously, $0<(2-\lambda) /(1-2 K \theta)-1<1$. The sequence $\left\{x_{n}\right\}$ converges to the exact solution $x_{*}$ from (32).
When $1<\lambda<2$, from (24),

$$
\begin{aligned}
& \left\|x_{n+1}-x_{*}\right\| \leqslant \frac{\left\|x_{n}-x_{*}\right\|}{1-K\left(\left\|x_{n}-x_{*}\right\|+\left\|x_{n-1}-x_{*}\right\|\right)} \\
& \quad \times\left(\lambda K \int _ { 0 } ^ { 1 } \left(\left\|t\left(x_{n-1}-x_{n}\right)+(1-t)\left(x_{n-1}-x_{*}\right)\right\|\right.\right. \\
& \left.+(1-t)\left\|x_{n}-x_{*}\right\|\right) \mathrm{d} t+(\lambda-1) \\
& \left.\cdot\left(K\left(\left\|x_{n-1}-x_{*}\right\|+\left\|x_{n}-x_{*}\right\|\right)+1\right)\right) .
\end{aligned}
$$

Then, by (27), we have

$$
\begin{aligned}
\theta_{n+1} & \leqslant \frac{\theta_{n}}{1-K\left(\theta_{n}+\theta_{n-1}\right)}\left(\lambda K\left(\theta_{n}+\theta_{n-1}\right)\right. \\
+ & \left.(\lambda-1)\left(K\left(\theta_{n}+\theta_{n-1}\right)+1\right)\right) \\
& =\frac{\theta_{n}}{1-K\left(\theta_{n}+\theta_{n-1}\right)}\left((2 \lambda-1) K\left(\theta_{n}+\theta_{n-1}\right)+\lambda\right.
\end{aligned}
$$$$
-1) \text {. }
$$

By (24) and (26), we know $\theta_{n}<r_{\lambda_{2}}$ for all $n$. Then by (26) and (34), when $1<\lambda<2$, we can induct

$$
\theta_{n+1}<\theta_{n}<\theta_{n-1}<\cdots<\theta_{1}<\theta
$$

So we have

$$
\begin{aligned}
\theta_{n+1} & \leqslant \theta_{n}\left(\frac{2(2 \lambda-1) K \theta-1}{1-2 K \theta}\right) \\
& <\theta\left(\frac{(4 \lambda-2) K \theta+\lambda-1}{1-2 K \theta}\right)^{n+1} .
\end{aligned}
$$

It is easy to proof that $0<((4 \lambda-2) K \theta+\lambda-1) /(1-2 K \theta)<1$. So the sequence $\left\{x_{n}\right\}$ converges to the solution $x_{*}$.

Now we show the uniqueness. Assume that there exists another solution $y_{*} \in B\left(x_{*}, 1 / K\right)$. Consider the operator $A=$ $\left[x_{*}, y_{*} ; F\right]$. Because $A\left[y_{*}-x_{*}\right]=F\left(y_{*}\right)-F\left(x_{*}\right)$, we can get $y_{*}=x_{*}$ if the operator $A$ is invertible. From (4), we get

$$
\begin{aligned}
\left\|I-F^{\prime}\left(x_{*}\right)^{-1} A\right\| & =\left\|F^{\prime}\left(x_{*}\right)^{-1}\left(F^{\prime}\left(x_{*}\right)-A\right)\right\| \\
& \leqslant K\left\|y_{*}-x_{*}\right\|<1 .
\end{aligned}
$$

So, we can tell that operator $A$ is invertible by Banach lemma. From the definition of $r_{\lambda}$ and (9), it is easy to verify that ball $B\left(x_{*}, 1 / K\right)$ is bigger than ball $B\left(x_{*}, r_{\lambda_{1}}\right)$ and ball $B\left(x_{*}, r_{\lambda_{2}}\right)$. Proof completes.

Remark 2. When $\lambda=1$, the radius of the convergence ball is $1 / 4 K$. We denote $r_{1}=1 / 4 K$. From (9), we know when $0<$ $\lambda<1, r_{\lambda_{1}}<r_{1}$, and when $1<\lambda<2, r_{\lambda_{2}}<r_{1}$. So we have the biggest convergence ball when $\lambda=1$.

\section{Numerical Examples}

In this section, we applied the convergence ball result given in Section 2 to solve some numerical problems. 
TABLE 1: Relaxed secant method with different $\lambda$.

\begin{tabular}{ccccc}
\hline$\lambda$ & $n$ & $x_{n}$ & $\left\|x_{n}-x_{*}\right\|$ & CPU time \\
\hline \multirow{4}{*}{0.9} & 1 & 1.0315 & 0.0315 & \\
& 2 & 1.0057 & 0.0057 & \\
& 3 & 1.0006 & $6.4804 \times 10^{-4}$ & 0.000877 \\
& 4 & 1.0001 & $6.6458 \times 10^{-5}$ & \\
& 5 & 1.0000 & $6.6652 \times 10^{-6}$ & \\
& 6 & 1.0000 & $6.6672 \times 10^{-7}$ & \\
& 1 & 1.0128 & 0.0128 & \\
& 2 & 1.0012 & 0.0012 & 0.000864 \\
& 3 & 1.0000 & $7.3141 \times 10^{-6}$ & \\
\hline \multirow{3}{*}{1.1} & 4 & 1.0000 & $4.2172 \times 10^{-7}$ & \\
& 1 & 0.9940 & 0.006 & \\
& 2 & 1.0000 & $1.6176 \times 10^{-6}$ & 0.000095 \\
\hline
\end{tabular}

Example 1. Let us consider

$$
F(x)=x^{2}-1, \quad x \in[0,2] .
$$

Then $F^{\prime}(x)=2 x . F(x)=0$ has a root $x_{*}=1$ and $F^{\prime}\left(x_{*}\right)=2$. It is easy to obtain

$$
\begin{gathered}
\left\|F^{\prime}\left(x_{*}\right)^{-1}([x, y ; F]-[u, v ; F])\right\| \\
\leqslant \frac{1}{2}(\|x-u\|+\|y-v\|) .
\end{gathered}
$$

Set $\lambda_{1}=0.9, \lambda_{2}=1, \lambda_{3}=1.1$. Then the radius of the convergence balls is $r_{1}=9 / 20, r_{2}=1 / 2, r_{3}=9 / 22$. Choose the initial points $x_{-1}=1.15, x_{0}=1.2$ and they are in the convergence ball of the relaxed secant method. From Table 1 , we can see the sequence $\left\{x_{n}\right\}$ converges to $x_{*}$ with different $\lambda$.

As we know, when $\lambda=1$, the relaxed secant method reduces to normal secant method. From Table 1, we can see that relaxed secant method in the case of $\lambda=1.1$ outperforms the normal secant method in the sense of iteration number and CPU time.

Example 2. Let us consider the following numerical problem which has been studied in $[3,17,18]$ :

$$
\begin{aligned}
F(x) & =e^{x}-1, \\
D & =[-1,1] .
\end{aligned}
$$

Then $F^{\prime}(x)=e^{x}, x_{*}=0$, and $F^{\prime}\left(x_{*}\right)=1$.

Similar to the process in [17], we know $\left|e^{x}-e^{y}\right| \leqslant e|x-y|$. Then,

$$
\left|\int_{0}^{1}\left(e^{t x+(1-t) y}-e^{t u+(1-t) v}\right) d t\right| \leqslant \frac{e}{2}(|x-u|+|y-v|) .
$$

For $[x, y ; F]=\int_{0}^{1} e^{t x+(1-t) y} \mathrm{~d} t$ and $\| F^{\prime}\left(x_{*}\right)^{-1}([x, y ; F]-[u, v$; $F])\|\leqslant\|[x, y ; F]-[u, v ; F] \|$, we can get

$$
\begin{gathered}
\left\|F^{\prime}\left(x_{*}\right)^{-1}([x, y ; F]-[u, v ; F])\right\| \\
\leqslant \frac{e}{2}(\|x-u\|+\|y-v\|) .
\end{gathered}
$$

TABLE 2: Relaxed secant method with different $\lambda$.

\begin{tabular}{lcccc}
\hline$\lambda$ & $n$ & $x_{n}$ & $\left\|x_{n}-x_{*}\right\|$ & CPU time \\
\hline \multirow{4}{*}{0.999} & 1 & 0.0038 & 0.0038 & \\
& 2 & $1.8245 \times 10^{-4}$ & $1.8245 \times 10^{-4}$ & 0.000094 \\
& 3 & $1.6290 \times 10^{-7}$ & $1.6290 \times 10^{-7}$ & \\
\hline \multirow{3}{*}{1} & 1 & 0.0039 & 0.0039 & \\
& 2 & $1.9078 \times 10^{-4}$ & $1.9078 \times 10^{-4}$ & 0.000094 \\
& 3 & $3.7011 \times 10^{-4}$ & $3.7011 \times 10^{-4}$ & \\
\hline \multirow{3}{*}{1.01} & 1 & 0.0048 & 0.0048 & \\
& 2 & $2.8301 \times 10^{-4}$ & $2.8301 \times 10^{-4}$ & 0.000108 \\
& 3 & $3.4788 \times 10^{-6}$ & $3.4788 \times 10^{-6}$ & \\
\hline
\end{tabular}

So $K=e / 2$ in this problem. Set $\lambda_{1}=0.999, \lambda_{2}=$ $1, \lambda_{3}=1.01$. Then, the radius of the convergence balls is $r_{1}=999 / 2000 e, r_{2}=1 / 2 e, r_{3}=99 / 202 e$. Set the initial points $x_{-1}=0.08, x_{0}=0.1$, and they are in the convergence ball of the relaxed secant method. From Table 2, we can see the sequence $\left\{x_{n}\right\}$ converges to the solution $x_{*}$.

From Table 2, we can know that the relaxed scant method $(\lambda=0.999)$ performs the same as the normal secant method in the sense of the iteration number and CPU time, while the solution gotten by the relaxed secant method is closer to the exact solution than that by the normal secant method.

Example 3. Let us consider the nonlinear system:

$$
\begin{aligned}
2 x_{1}-\frac{1}{9} x_{1}^{2}-x_{2} & =0 \\
-x_{1}+2 x_{2}-\frac{1}{9} x_{2}^{2} & =0
\end{aligned}
$$

It comes from the following nonlinear boundary value problem of second order:

$$
\begin{aligned}
x^{\prime \prime}+x^{2} & =0, \\
x(0) & =x(1)=0,
\end{aligned}
$$

which has been studied by many authors $[5,13,16]$.

Now, define the operator $F: R^{2} \rightarrow R^{2}$ such that $F=\left(F_{1}, F_{2}\right)$. We take $F_{1}\left(x_{1}, x_{2}\right)=2 x_{1}-(1 / 9) x_{1}^{2}-x_{2}=$ $0, F_{2}\left(x_{1}, x_{2}\right)=-x_{1}+2 x_{2}-(1 / 9) x_{2}^{2}=0, x=\left(x_{1}, x_{2}\right) \in R^{2}$. Then, notice $0<\lambda \leqslant 1$; it is easy to know $F$ is Fréchet differentiable in $R^{2}$ and we get

$$
F^{\prime}(x)=\left(\begin{array}{cc}
2-\frac{2}{9} x_{1} & -1 \\
-1 & 2-\frac{2}{9} x_{2}
\end{array}\right)
$$

Let $x=\left(x_{1}, x_{2}\right) \in R^{2}$ and $\|x\|=\|x\|_{\infty}=\max _{1 \leqslant i \leqslant 2}\left|x_{i}\right|$. The corresponding norm on $A \in R^{2} \times R^{2}$ is

$$
\|A\|=\max _{1 \leqslant i \leqslant 2} \sum_{j=1}^{2}\left|a_{i j}\right| .
$$


TABLE 3: Relaxed secant method with different $\lambda$.

\begin{tabular}{llccc}
\hline$\lambda$ & $n$ & $x_{n}$ & $\left\|x_{n}-x_{*}\right\|$ & CPU time \\
\hline \multirow{4}{*}{0.9999} & 1 & $(8.9732,8.9732)$ & 0.0277 & \\
& 2 & $(9.0016,9.0016)$ & 0.0016 & 0.000288 \\
& 3 & $(9.0000,9.0000)$ & $5.2307 \times 10^{-6}$ & \\
\hline \multirow{4}{*}{1} & 4 & $(9.0000,9.0000)$ & $4.2965 \times 10^{-10}$ & \\
& 1 & $(8.9722,8.9722)$ & 0.0278 & \\
& 2 & $(9.0016,9.0016)$ & 0.0016 & 0.001643 \\
& 3 & $(9.0000,9.0000)$ & $5.0744 \times 10^{-6}$ & \\
& 4 & $(9.0000,9.0000)$ & $9.2414 \times 10^{-10}$ & \\
\hline \multirow{3}{*}{0.8} & 1 & $(8.9455,8.9455)$ & 0.545 & \\
& 2 & $(9.0000,9.0000)$ & $4.0974 \times 10^{-5}$ & 0.001608 \\
& 3 & $(9.0000,9.0000)$ & $2.0838 \times 10^{-6}$ & \\
\hline & 4 & $(9.0000,9.0000)$ & $1.1794 \times 10^{-7}$ & \\
\hline
\end{tabular}

It can be verified easily that $x_{*}=(9,9)$ is a solution of (24) and from (26) we get

$$
F^{\prime}(x)=\left(\begin{array}{cc}
0 & -1 \\
-1 & 0
\end{array}\right) .
$$

$F^{\prime}(x)$ is invertible. Similar to [13], we can deduce that Lipschitz continuous condition is satisfied for $K=1 / 9$. Set $\lambda_{1}=0.9999, \lambda_{2}=1, \lambda_{3}=1.06$. Then the radius of the convergence ball is $r_{1}=2.499975, r_{2}=9 / 4, r_{3}=326 / 212$. Set the two initial points $x_{-1}=(9.5,9.5), x_{0}=(8.5,8.5)$ and they are in the convergence ball. For results, see Table 3 .

Table 3 shows the sequence $\left\{x_{n}\right\}$ generated by the relaxed secant method. From this table, it is known that the sequence $\left\{x_{n}\right\}$ converges, and also the error estimation holds. Moreover, relaxed secant method has more choices than secant method, and optimal parameter $\lambda$ makes the presented method outperforms the normal secant method.

Example 4. Consider the nonlinear conservative system given in [15]:

$$
\begin{aligned}
\frac{d^{2} x(t)}{d t^{2}} & =-e^{x(t)}, \\
x(0) & =x(1)=0 .
\end{aligned}
$$

Applying the centered finite difference scheme, we can get the nonlinear system:

$$
F(x)=M x+h^{2} \phi(x),
$$

where $h=1 /(N+1)$ is the step-size and $N$ is a prescribed positive integer. $x, \phi(x)$ are vectors with forms of

$$
\begin{gathered}
x=\left(\begin{array}{c}
x_{1} \\
x_{2} \\
\vdots \\
x_{m}
\end{array}\right), \\
\phi(x)=\left(\begin{array}{c}
e^{x_{1}} \\
e^{x_{2}} \\
\vdots \\
e^{x_{m}}
\end{array}\right),
\end{gathered}
$$

TABLE 4: Numerical results for nonlinear conservative systems.

\begin{tabular}{llll}
\hline$\lambda$ & IT & $\left\|x_{n+1}-x_{n}\right\|$ & CPU time \\
\hline 0.99 & 75 & $8.7445 \times 10^{-7}$ & 0.002581 \\
1 & 94 & $1.9319 \times 10^{-7}$ & 0.002931 \\
0.001 & 63 & $9.3315 \times 10^{-7}$ & 0.001471 \\
\hline
\end{tabular}

TABLE 5: Approximated solution.

\begin{tabular}{lc}
\hline$i$ & $x_{i}^{*}$ \\
\hline 1 & 0.026205377 \\
2 & 0.049844664 \\
3 & 0.070856372 \\
4 & 0.089184975 \\
5 & 0.104780806 \\
6 & 0.117601571 \\
7 & 0.127610864 \\
8 & 0.134780833 \\
9 & 0.139090798 \\
10 & 0.140529159 \\
11 & 0.139090906 \\
12 & 0.134780768 \\
13 & 0.127610728 \\
14 & 0.117601588 \\
15 & 0.104780816 \\
16 & 0.089184869 \\
17 & 0.070856314 \\
18 & 0.049844674 \\
19 & 0.026205359 \\
\hline
\end{tabular}

and the matrix $M$ has the form

$$
A=\left(\begin{array}{ccccc}
-2 & 1 & 0 & \cdots & 0 \\
1 & -2 & 1 & \cdots & 0 \\
0 & 1 & -2 & \cdots & 0 \\
\vdots & \vdots & \vdots & \ddots & \vdots \\
0 & 0 & 0 & \cdots & -2
\end{array}\right) .
$$

Take the same parameters used in [15], $N=19, h=1 / 20$, and the initial points $x_{-1}(t)=(5 / 2) t(1-t)$ and $x_{0}(t)=$ $(1 / 2) t(1-t), t \in[0,1]$. Then, we can solve this problem by our relaxed secant method, and we compare it with normal secant method. For the results, see Table 4.

From the results, we can know that, in this example, the relaxed secant method performs better. And we list the approximation solution which is gotten by the relaxed secant method in the situation $\lambda=0.99$ in Table 5 .

\section{Competing Interests}

The authors declare that they have no competing interests. 


\section{Acknowledgments}

This work is supported by National Natural Science Foundation of China (Grant nos. 11371320, 11632015), Zhejiang Natural Science Foundation (Grant no. LZ14A010002), and Scientific Research Fund of Zhejiang Provincial Education Department (Grant no. FX2016073).

\section{References}

[1] I. K. Argyros, "A new semilocal convergence theorem for Newton's method in Banach space using hypotheses on the second Fréchet-derivative," Journal of Computational and Applied Mathematics, vol. 130, no. 1-2, pp. 369-373, 2001.

[2] I. K. Argyros, "On the Newton-Kantorovich hypothesis for solving equations," Journal of Computational and Applied Mathematics, vol. 169, no. 2, pp. 315-332, 2004.

[3] I. K. Argyros and S. Hilout, "On the local convergence of fast two-step Newton-like methods for solving nonlinear equations," Journal of Computational and Applied Mathematics, vol. 245, pp. 1-9, 2013.

[4] M. A. Hernández and M. J. Rubio, "A modifcation of Newton's method for nondiferentiable equations," Journal of Computational and Applied Mathematics, vol. 164, pp. 409-417, 2004.

[5] M. A. Hernández and M. J. Rubio, "A uniparametric family of iterative processes for solving nondifferentiable equations," Journal of Mathematical Analysis and Applications, vol. 275, no. 2, pp. 821-834, 2002.

[6] M. T. Darvishi, "A two-step high order Newton-like method for solving systems of nonlinear equations," International Journal of Pure and Applied Mathematics, vol. 57, no. 4, pp. 543-555, 2009.

[7] S. Amat, A. Magreñán, and N. Romero, "On a two-step relaxed Newton-type method," Applied Mathematics and Computation, vol. 219, no. 24, pp. 11341-11347, 2013.

[8] I. K. Argyros and Á. A. Magreñán, "On the convergence of inexact two-point Newton-like methods on Banach spaces," Applied Mathematics and Computation, vol. 265, Article ID 21248, pp. 893-902, 2015.

[9] I. K. Argyros, A. Cordero, A. Magreñán, and J. R. Torregrosa, "On the convergence of a damped Newton-like method with modified right hand side vector," Applied Mathematics and Computation, vol. 266, Article ID 21280, pp. 927-936, 2015.

[10] J. A. Ezquerro, D. González, and M. A. Hernández-Verón, "A semilocal convergence result for Newton's method under generalized conditions of Kantorovich," Journal of Complexity, vol. 30, no. 3, pp. 309-324, 2014.

[11] O. P. Ferreira, "A robust semi-local convergence analysis of Newton's method for cone inclusion problems in Banach spaces under affine invariant majorant condition," Journal of Computational and Applied Mathematics, vol. 279, pp. 318-335, 2015.

[12] I. K. Argyros and S. George, "Unified convergence domains of Newton-like methods for solving operator equations," Applied Mathematics and Computation, vol. 286, pp. 106-114, 2016.

[13] H. M. Ren and Q. B. Wu, "The convergence ball of the Secant method under Hölder continuous divided differences," Journal of Computational and Applied Mathematics, vol. 194, no. 2, pp. 284-293, 2006.

[14] I. K. Argyros and S. K. Khattri, "On the Secant method," Journal of Complexity, vol. 29, no. 6, pp. 454-471, 2013.
[15] J. A. Ezquerro, M. Grau-Sánchez, M. A. Hernández, and M. Noguera, "Semilocal convergence of secant-like methods for differentiable and nondifferentiable operator equations," Journal of Mathematical Analysis and Applications, vol. 398, no. 1, pp. 100-112, 2013.

[16] M. A. Hernandez and M. J. Rubio, "The secant method and divided differences Holder continuous," Applied Mathematics and Computation, vol. 124, no. 2, pp. 139-149, 2001.

[17] H. M. Ren, S. J. Yang, and Q. B. Wu, "A new semilocal convergence theorem for the Secant method under Hölder continuous divided differences," Applied Mathematics and Computation, vol. 182, no. 1, pp. 41-48, 2006.

[18] H. M. Ren and Q. B. Wu, "Mysovskii-type theorem for the secant method under Hölder continuous Fréchet derivative," Journal of Mathematical Analysis and Applications, vol. 320, no. 1, pp. 415-424, 2006. 


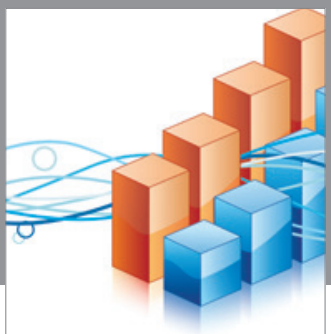

Advances in

Operations Research

vatem alat4

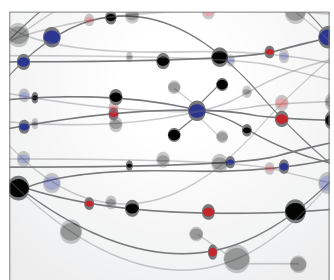

\section{The Scientific} World Journal
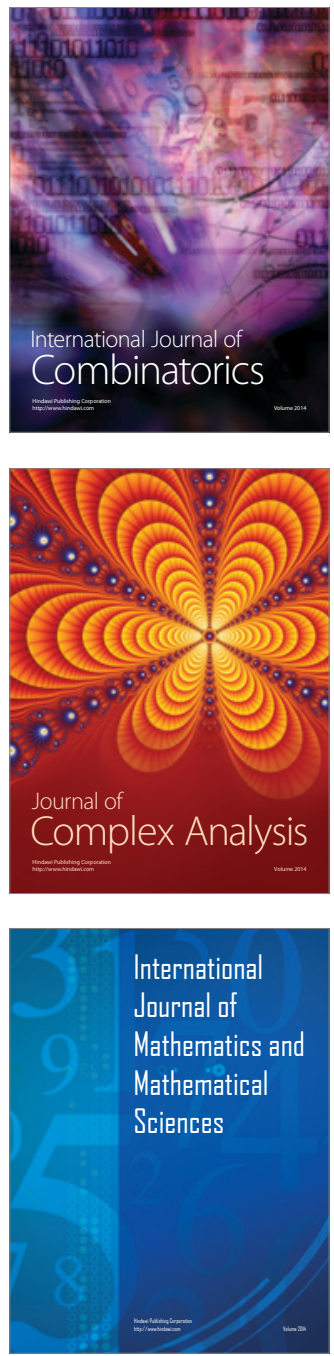
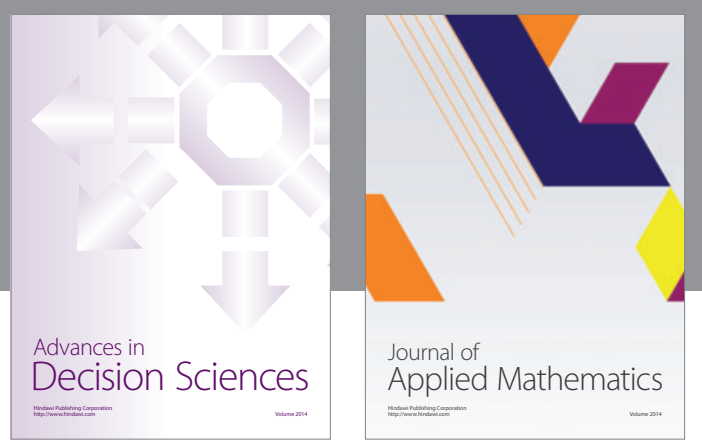

Algebra

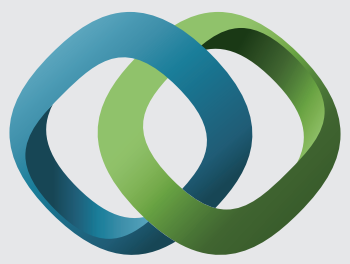

\section{Hindawi}

Submit your manuscripts at

https://www.hindawi.com
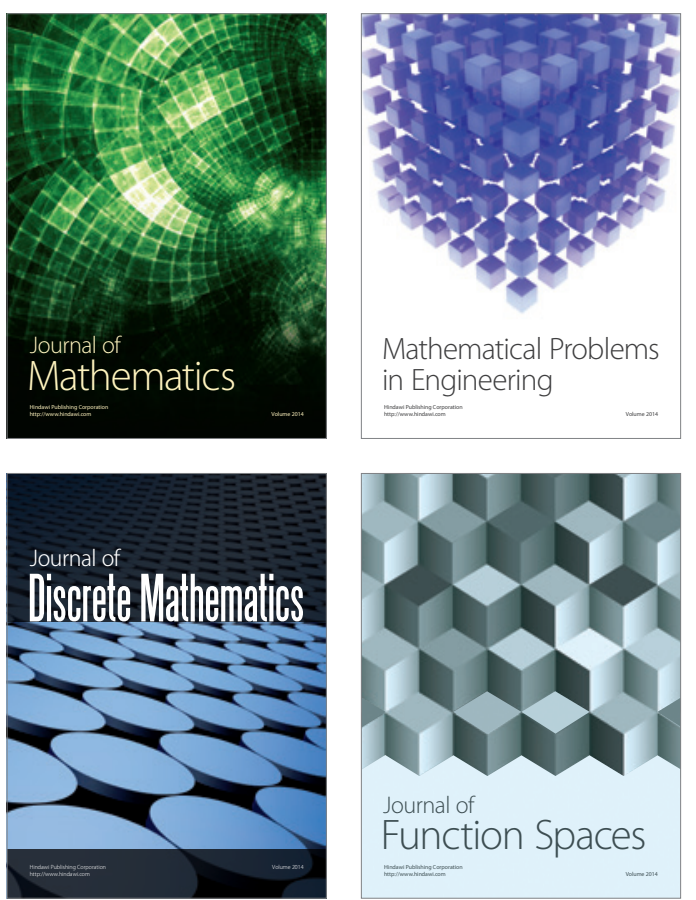

Mathematical Problems in Engineering
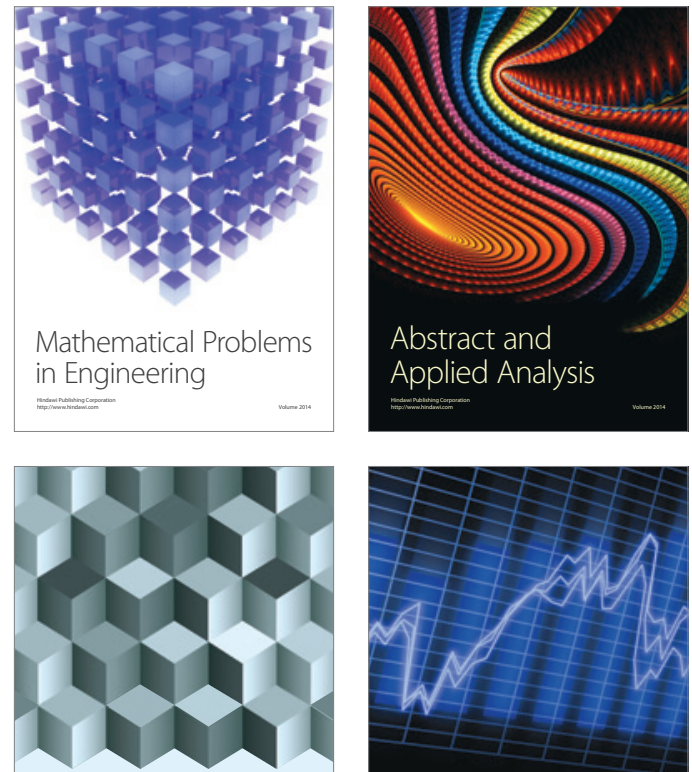

Journal of

Function Spaces

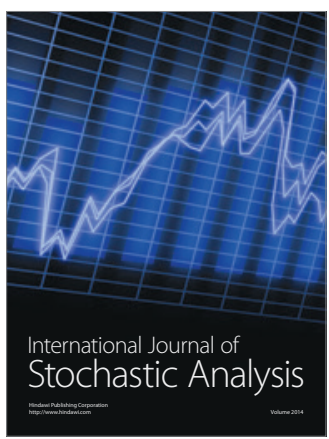

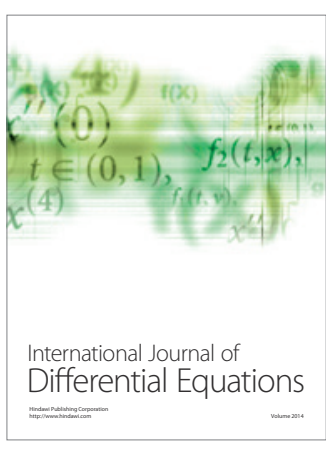
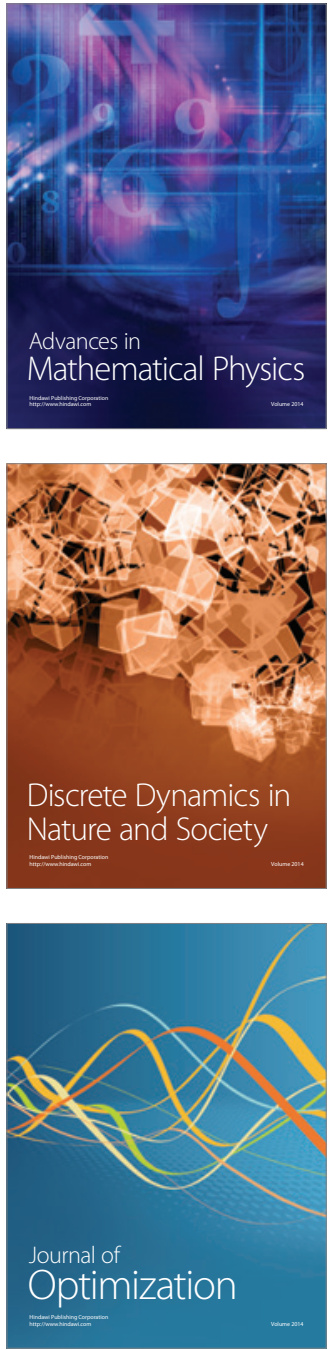